\title{
Quantitative Dietary Fingerprinting (QDF) - A Novel Tool for Comprehensive Dietary Assessment Based on Urinary Nutrimetabolomics
}

\author{
Raúl González-Domínguez, ${ }^{\dagger, \uparrow}$ Mireia Urpi-Sarda, ${ }^{\dagger}{ }^{\ddagger}$ Olga Jáuregui, ${ }^{\ddagger}{ }^{\ddagger}$ Paul W. Needs, $"$ \\ Paul A. Kroon, ${ }^{\| \odot}$ and Cristina Andrés-Lacueva* ${ }^{\dagger}, \ddagger \odot$
}

\begin{abstract}
${ }^{\dagger}$ Biomarkers and Nutrimetabolomics Laboratory, Department of Nutrition, Food Sciences and Gastronomy, Food Technology Reference Net (XaRTA), Nutrition and Food Safety Research Institute (INSA), Faculty of Pharmacy and Food Sciences, University of Barcelona, 08028 Barcelona, Spain

${ }^{\ddagger}$ CIBER Fragilidad y Envejecimiento Saludable (CIBERfes), Instituto de Salud Carlos III, 08028 Barcelona, Spain

${ }^{\S}$ Scientific and Technological Center of University of Barcelona (CCiTUB), 08028 Barcelona, Spain

"Quadram Institute Bioscience, Norwich Research Park, Norwich NR4 7UA, United Kingdom
\end{abstract}

Supporting Information

ABSTRACT: Accurate dietary assessment is a challenge in nutritional research, needing powerful and robust tools for reliable measurement of food intake biomarkers. In this work, we have developed a novel quantitative dietary fingerprinting (QDF) approach, which enables for the first time the simultaneous quantitation of about 350 urinary food-derived metabolites, including (poly)phenolic aglycones, phase II metabolites, and microbial-transformed compounds, as well as other compounds (e.g., glucosinolates, amino acid derivatives, methylxanthines, alkaloids, and markers of alcohol and tobacco consumption). This method was fully validated for 220 metabolites, yielding good linearity, high sensitivity and precision, accurate recovery rates, and negligible matrix effects. Furthermore, 127 additional phase II metabolites were also included in this method after identification in urines collected from acute dietary interventions with various foods. Thus, this metabolomic approach represents one-step further toward precision nutrition and the objective of improving the accurateness and comprehensiveness in the assessment of dietary patterns and lifestyles.

KEYWORDS: quantitative dietary fingerprinting (QDF), targeted metabolomics, dietary assessment, ultra-high-performance liquid chromatography-tandem mass spectrometry, urine

\section{INTRODUCTION}

Accurate assessment of food intake is a keystone in nutritional research, with the aim of monitoring dietary patterns in freeliving populations, understanding the association between nutrition and health status, and ensuring compliance in dietary intervention studies. This goal has traditionally been accomplished using self-reporting methods, namely, food frequency questionnaires, dietary recalls, or diet diaries. A major limitation of these tools is the high prevalence of misreporting as a result of their subjective nature. ${ }^{1}$ On the other hand, the measurement of metabolomic dietary biomarkers has emerged in recent years as a more reliable approach for characterizing the nutritional status. ${ }^{2}$ In this context, the food metabolome can be defined as the entire set of metabolites coming from the ingestion, absorption, and biotransformation of food components, comprising a myriad of different compounds, including polyphenols, carotenoids, glucosinolates, amino acids and derived metabolites, fatty acids, and many others. ${ }^{3}$ In particular, (poly)phenolic compounds constitute a diverse group of secondary metabolites widely distributed in the plant kingdom, and these have been proposed as suitable biomarkers of fruit and vegetable consumption. ${ }^{4}$ After ingestion, polyphenols undergo extensive metabolization by phase I and II enzymes, yielding glucuronidated, sulfated, and methylated metabolites. ${ }^{5}$ However, numerous polyphenols are scarcely absorbed in the small intestine as a result of their low bioavailability; therefore, they are metabolized in the colon by gut microbiota into phenolic acids and other easily absorbable metabolites, which, in turn, can also be conjugated to their glucuronide and sulfate forms. ${ }^{6}$ Thus, original food compounds are usually detected in the organism at very low concentration levels compared to their biotransformed metabolites. Given this complexity of the food metabolome, analytical methods with wide coverage and high sensitivity are needed to assess the metabolic fingerprint of foods and dietary patterns by monitoring the large number of potential dietary biomarkers in biological samples. To this end, targeted metabolomics based on mass spectrometry is a very attractive strategy because it enables sensitive and specific quantification of metabolites. ${ }^{7}$ The majority of previously published studies in this field are based on the use of an enzymatic hydrolysis step with the aim of estimating the

Special Issue: Food Bioactives and Health

Received: December 17, 2018

Revised: February 23, 2019

Accepted: February 25, 2019

Published: February 25, 2019 
concentrations of glucuronide and sulfate derivatives as aglycone equivalents because of the lack of commercial standards for many of these phase II metabolites. ${ }^{8}$ However, this approach has important drawbacks, such as the loss of relevant information about the chemical nature of phenolic conjugates, the introduction of impurities from commercially available enzyme preparations, and the underestimation of polyphenol concentrations as a result of incomplete hydrolysis and low recovery of free forms. ${ }^{9-11}$ Another proposed strategy is the analysis of phase II metabolites using calibration curves of the corresponding aglycones, which provides inaccurate results as a result of the assumption that chromatographic and mass spectrometric responses of conjugated compounds are equivalent to those shown by their aglycones. ${ }^{12,13}$ However, only a few validated methods have been reported for direct quantification of phase II metabolites, with most of them focused on specific phenolic classes. ${ }^{14-17}$

The aim of this work was the development and validation of a quantitative targeted urinary nutrimetabolomic platform to assess dietary patterns and lifestyle, termed as quantitative dietary fingerprinting (QDF). To this end, solid-phase extraction (SPE) was combined with ultra-high-performance liquid chromatography coupled to tandem mass spectrometry (UHPLC-MS/MS), which enabled the simultaneous quantitation of almost 350 dietary markers, including (poly)phenolic aglycones, phase II metabolites, microbial-transformed compounds, and other dietary components.

\section{MATERIALS AND METHODS}

Chemicals and Urine Samples. The provenance of chemicals and standards is detailed in the Supporting Information. Blank human urine samples were collected from healthy volunteers after 1 week of a low-polyphenol diet. ${ }^{15}$ Various dietary interventions were also conducted to obtain urine samples enriched in food metabolites with the aim of (a) looking for potential biomarkers for which standards are not available and (b) applying the validated methodology to demonstrate its utility. For this purpose, healthy volunteers $(n=3)$ were asked to follow a low polyphenol diet 2 days before the interventions and then to supplement their diet by consuming the following foods: orange, grapefruit, apple, red wine, beer, green tea, coffee, soy sprouts, walnuts, and whole grain bread (detailed information in Table S1 of the Supporting Information). These foods were consumed at dinner, and then participants collected first morning void urine samples. Samples were aliquoted and stored at $-80{ }^{\circ} \mathrm{C}$ until analysis. The study was performed in accordance with the principles contained in the Declaration of Helsinki. The Bioethical Committee of the University of Barcelona approved the study protocol, and all of the participants provided written informed consent. The study was inscribed as ISRCTN17200423 in the International Standard Randomized Controlled Trial Number registry.

Sample Treatment. Urine samples were thawed in an ice bath and then centrifuged at $10000 \mathrm{~g}$ for $10 \mathrm{~min}$ at $4{ }^{\circ} \mathrm{C}$. Then, different sample treatment procedures were compared, including dilution and two complementary SPE devices, Oasis HLB and Oasis MAX 96-well plates, filled with $30 \mathrm{mg}$ of sorbent (Waters, Milford, MA, U.S.A.). For SPE extraction, a Waters Positive Pressure-96 Processor was employed.

Urine Dilution. A $50 \mu \mathrm{L}$ aliquot of the previously centrifuged urine was diluted with $50 \mu \mathrm{L}(1: 1, \mathrm{v} / \mathrm{v}), 450 \mu \mathrm{L}(1: 10, \mathrm{v} / \mathrm{v})$, or $950 \mu \mathrm{L}$ $(1: 100, \mathrm{v} / \mathrm{v})$ of ultrapure water containing $0.1 \%$ formic acid $(\mathrm{v} / \mathrm{v})$. Taxifolin was added as an internal standard (IS) to reach a final concentration of $100 \mu \mathrm{g} \mathrm{L}^{-1}$, and samples were transferred to 96-well plates for further analysis.

SPE (MAX-Mixed-Mode Sorbent). SPE using the Oasis MAX plate was performed following the method described by Zamora-Ros et al., with some modifications. ${ }^{18}$ Briefly, the sorbent first had to be conditioned with methanol and $50 \mathrm{mM}$ sodium acetate $(\mathrm{pH} 7)$. Then, $1 \mathrm{~mL}$ of urine containing $\mathrm{H}_{3} \mathrm{PO}_{4}(2 \%, \mathrm{v} / \mathrm{v})$ and $100 \mu \mathrm{g} \mathrm{L}{ }^{-1}$ of a set of isotopically labeled ISs (ferulic acid-1,2,3 $-{ }^{13} \mathrm{C}_{3}, p$-coumaric acid$1,2,3-{ }^{13} \mathrm{C}_{3}$, catechin- $2,3,4-{ }^{13} \mathrm{C}_{3}$, and L-phenylalanine- ${ }^{15} \mathrm{~N}$ ) was loaded onto the pre-conditioned plate. Plates were washed with $5 \%$ methanol in $50 \mathrm{mM}$ sodium acetate ( $\mathrm{pH} \mathrm{7)}$ ), and analytes were eluted with $2 \%$ formic acid in methanol $(\mathrm{v} / \mathrm{v})$. Finally, extracts were taken to dryness using a MaxiVac $\beta$ vacuum concentrator (Daejeon, South Korea) and then reconstituted with $100 \mu \mathrm{L}$ of taxifolin $\left(100 \mu \mathrm{g} \mathrm{L}^{-1}\right)$ dissolved in the mobile phase as the IS.

SPE (HLB-Reversed-Phase Sorbent). Three extraction protocols based on the use of Oasis HLB plates were compared. (i) First, we applied the method developed by Urpi-Sarda et al., which employs methanol and $1.5 \mathrm{M}$ formic acid in water for SPE cartridge conditioning. ${ }^{19}$ After acidulated urine $\left(2 \%, \mathrm{v} / \mathrm{v}, \mathrm{H}_{3} \mathrm{PO}_{4}\right)$ was loaded, spiked with the set of isotopically labeled ISs previously described, cleanup was performed with $1.5 \mathrm{M}$ formic acid and 5\% methanol in water. Finally, retained metabolites were eluted with methanol containing $0.1 \%$ formic acid. (ii) To improve the extraction of sulfate metabolites, a slight modification of a previously described method was also tested on the basis of the application of a two-step elution process with $0.5 \mathrm{~mL}$ of $0.1 \%$ ammonia in methanol and $1 \mathrm{~mL}$ of $0.1 \%$ formic acid in methanol. ${ }^{20}$ (iii) In the same vein, we also evaluated the utility of adding ammonium formate to SPE solvents to increase extraction recoveries. For this, plates were conditioned with $1 \mathrm{~mL}$ of methanol and $1 \mathrm{~mL}$ of water containing $0.1 \%$ formic acid and $10 \mathrm{mM}$ ammonium formate. Then, $1 \mathrm{~mL}$ of acidulated urine containing the set of primary ISs was loaded, followed by $1 \mathrm{~mL}$ of water containing $0.1 \%$ formic acid and $10 \mathrm{mM}$ ammonium formate. Finally, elution was accomplished with $1.5 \mathrm{~mL}$ of methanol containing $0.1 \%$ formic acid and $10 \mathrm{mM}$ ammonium formate. All of these extracts were taken to dryness using a vacuum concentrator and were then reconstituted with $100 \mu \mathrm{L}$ of taxifolin $\left(100 \mu \mathrm{g} \mathrm{L}^{-1}\right)$ dissolved in the mobile phase as the IS.

UHPLC-MS/MS Analysis. Targeted metabolomic profiling of the urinary food metabolome was carried out on an Agilent 1290 Infinity UHPLC system (Santa Clara, CA, U.S.A.) coupled to a Sciex QTRAP 6500 mass spectrometer equipped with Ion Drive Turbo $\mathrm{V}$ ion source (Framingham, MA, U.S.A.). The column used was a $100 \times 2.1 \mathrm{~mm}$ (1.6 $\mu \mathrm{m}$ inner diameter) Luna Omega Polar C18, equipped with a fully porous polar C18 security guard cartridge from Phenomenex (Torrance, CA, U.S.A.). Chromatographic conditions were as follows: column temperature, $40{ }^{\circ} \mathrm{C}$; autosampler temperature, $4{ }^{\circ} \mathrm{C}$; injection volume, $2 \mu \mathrm{L}$; and flow rate, $0.5 \mathrm{~mL} \mathrm{~min}^{-1}$. Two different mobile phase combinations were employed depending upon the ionization mode of data acquisition. In the negative ion mode, $0.1 \%$ formic acid and $10 \mathrm{mM}$ ammonium formate in water and pure acetonitrile were delivered as aqueous (A) and organic (B) mobile phases, respectively. The gradient program was as follows: $0-8 \mathrm{~min}, 5-20 \% \mathrm{~B} ; 8-10 \mathrm{~min}$, $20-100 \% \mathrm{~B} ; 10-12 \mathrm{~min}, 100 \% \mathrm{~B} ; 12-12.1 \mathrm{~min}, 100-5 \% \mathrm{~B}$; and 12.1-14 $\mathrm{min}, 5 \% \mathrm{~B}$. On the other hand, water and acetonitrile, both containing $0.5 \%$ formic acid, were used as mobile phases when the mass spectrometer operated in the positive ion mode. In that case, the gradient profile was as follows: $0-5 \mathrm{~min}, 5-50 \% \mathrm{~B} ; 5-5.1 \mathrm{~min}, 50-$ $100 \% \mathrm{~B} ; 5.1-7 \mathrm{~min}, 100 \% \mathrm{~B}$; 7-7.1 $\mathrm{min}, 100-5 \% \mathrm{~B}$; and $7.1-9 \mathrm{~min}$, $5 \% \mathrm{~B}$.

MS detection was performed using the scheduled multiple reaction monitoring (sMRM) mode. The mass spectrometer operated in positive and negative ionization modes in separate runs, using the following parameters: ion spray voltage, $+5500 /-4000 \mathrm{~V}$; source temperature, $600{ }^{\circ} \mathrm{C}$; curtain gas, $30 \mathrm{psi}$; ion source gases 1 and 2, 50 psi each; collision-activated dissociation gas, $3 \mathrm{psi}$; and entrance potential, $( \pm) 10 \mathrm{~V}$. The multiple reaction monitoring (MRM) transitions were optimized by infusing individual solutions of commercial standards dissolved in the mobile phase $(1: 1, \mathrm{v} / \mathrm{v}$, proportion $\mathrm{A} / \mathrm{B}, 500 \mu \mathrm{g} \mathrm{L}^{-1}$ ) into the mass spectrometer using a syringe pump at a flow rate of $5 \mu \mathrm{L} \mathrm{min}{ }^{-1}$. The method also included some phase II metabolites identified in urine samples by product ion scan monitoring, for which authentic standards were not available. 


\section{Table 1. Metabolites Included in the QDF Approach as Food Intake Biomarkers}

\begin{tabular}{|c|c|c|}
\hline metabolite class & food & reference \\
\hline $\begin{array}{l}\text { hydroxybenzoic acids: derivatives of benzoic, hippuric, gallic, (iso)vanillic, and } \\
\text { syringic acids }\end{array}$ & $\begin{array}{l}\text { fruits and vegetables, tea, coffee, cocoa, grains, nuts, } \\
\text { legumes, olive oil, and wine }\end{array}$ & $22-29$ \\
\hline hydroxyphenylacetic acids: derivatives of phenylacetic and homovanillic acids & $\begin{array}{l}\text { fruits and vegetables, tea, cocoa, olives and olive oil, } \\
\text { and wine }\end{array}$ & $22-24,28$, and 29 \\
\hline hydroxycinnamic acids: derivatives of coumaric, caffeic, (iso)ferulic, and sinapic acids & $\begin{array}{l}\text { fruits and vegetables, coffee, cocoa, grains, nuts, } \\
\text { legumes, olive oil, and wine }\end{array}$ & $22-29$ \\
\hline $\begin{array}{l}\text { hydroxyphenylpropionic acids: derivatives of phenylpropionic, dihydrocaffeic, and } \\
\text { dihydro(iso)ferulic acids }\end{array}$ & $\begin{array}{l}\text { fruits and vegetables, coffee, tea, cocoa, grains, and } \\
\text { olive oil }\end{array}$ & $22-25$ and 28 \\
\hline flavan-3-ols: (epi)catechin derivatives & $\begin{array}{l}\text { fruits (berries and apple), tea, cocoa, nuts, legumes, } \\
\text { and wine }\end{array}$ & $\begin{array}{l}23,24,26,27,29 \text {, } \\
\text { and } 30\end{array}$ \\
\hline $\begin{array}{l}\text { hydroxyphenylvalerolactones: derivatives of phenylvaleric acid and } \\
\text { phenylvalerolactones (microbial metabolites of flavan-3-ols) }\end{array}$ & $\begin{array}{l}\text { procyanidin-rich foods (berries, apple, tea, cocoa, wine, } \\
\text { nuts, and legumes) }\end{array}$ & $\begin{array}{l}23,24,26,27,29 \text {, } \\
\text { and } 30\end{array}$ \\
\hline flavanones: derivatives of naringenin and hesperetin & citrus fruits and tomato (minor) & 31 \\
\hline isoflavones: derivatives of daidzein, genistein, equol, biochanin A, and formononetin & soy and legumes & 27 \\
\hline flavones: derivatives of apigenin and luteolin & fruits and vegetables, grains, olive oil, and legumes & 31 \\
\hline flavonols: derivatives of quercetin, kaempferol, and isorhamnetin & $\begin{array}{l}\text { fruits and vegetables, tea, wine, nuts, olive oil, and } \\
\text { legumes }\end{array}$ & 32 \\
\hline $\begin{array}{l}\text { anthocyanins: derivatives of cyanidin, peonidin, malvidin, pelargonidin, peonidin, and } \\
\text { petunidin }\end{array}$ & wine and berries & 29 \\
\hline dihydrochalcones: phloretin derivatives & apple & 30 \\
\hline stilbenes: resveratrol derivatives & wine & 29 \\
\hline phenylethanoids: tyrosol derivatives & olive oil & 28 \\
\hline lignans: derivatives of enterolactone and enterodiol (microbial metabolites) & $\begin{array}{l}\text { fiber-rich fruits and vegetables, grains, nuts, and } \\
\text { legumes }\end{array}$ & 32 \\
\hline prenylflavonoids: isoxanthohumol & beer & 33 \\
\hline coumarins: derivatives of bergaptol and umbelliferone & fruits (Rutaceae) and vegetables (Umbelliferae) & 34 \\
\hline curcuminoids: curcumin derivatives & Curcuma & 31 \\
\hline urolithins: microbial metabolites of ellagitannins & berries, wine, pomegranate, and nuts & 16 \\
\hline $\begin{array}{l}\text { other polyphenols: derivatives of pyrogallol, catechol, and hydroxybenzaldehydes } \\
\text { (microbial metabolites) }\end{array}$ & fruits and vegetables & 35 and 36 \\
\hline $\begin{array}{l}\text { benzoxazinoids: derivatives of aminophenol and hydroxyphenylacetamide (microbial } \\
\text { metabolites) }\end{array}$ & grains & 37 \\
\hline glucosinolates: sulforaphane derivatives & $\begin{array}{l}\text { cruciferous vegetables (cabbage, broccoli, and Brussels } \\
\text { sprouts) }\end{array}$ & 38 \\
\hline$S$-allylcysteine derivatives & Allium vegetables (garlic and onion) & 39 \\
\hline glycoalkaloids: $\alpha$-solanine, $\alpha$-chaconine, and solanidine & potato & 40 \\
\hline pyridinium derivatives: trigonelline and $N$-methylpyridinium & coffee and legumes & 41 \\
\hline $\begin{array}{l}\text { amino acid derivatives and endogenous: } 1 / 3 \text {-methylhistidine, carnosine, anserine, } \\
\text { L-carnitine, and creatinine }\end{array}$ & meat, fish, and dairy products & 42 and 43 \\
\hline methylxanthines: derivatives of xanthine and uric acid & coffee, tea, and cocoa & 23 and 24 \\
\hline diketopiperazines: cyclo(Leu-Pro) and cyclo(Pro-Val) & heat-treated foods (coffee and cocoa) & 23 and 24 \\
\hline furan derivatives (Maillard reaction products) & $\begin{array}{l}\text { heat-treated foods (coffee, grains, and fruits and } \\
\text { vegetables) }\end{array}$ & 44 \\
\hline polycyclic compounds: PhIP-G and 1-hydroxypyrene glucuronide & heat-treated meat and fish & 45 \\
\hline ethanol derivatives: ethyl glucuronide and sulfate & alcoholic beverages & 46 \\
\hline proline betaine and 4-hydroxyproline betaine & citrus fruits & 47 \\
\hline artificial sweeteners: acesulfame, cyclamate, and saccharin & sweetened beverages & 23 \\
\hline pyridine alkaloids: derivatives of nicotine and cotinine & smoking status & 48 \\
\hline others: TMAO, arsenobetaine, ergothioneine, tartaric acid, and hordenine & fish and seafood, mushrooms, wine, and beer & $33,44,49$, \\
\hline
\end{tabular}

Their MRM parameters were estimated on the basis of results obtained with structurally similar standards (see the Application to Real Urine Samples: Identification of Novel Conjugate Metabolites section). Because of the large number of compounds monitored in narrow retention time (RT) windows, the target scan time was set at $0.1 \mathrm{~s}$. Optimized MRM transitions, declustering potentials (DPs), collision energies (CEs), cell exit potentials (CXPs), RTs, and RT windows are listed in Table S2 of the Supporting Information. Analyst 1.6.2 and MultiQuant 3.0.3 software (ABSciex, Framingham, MA, U.S.A.) were used for data acquisition and data processing, respectively.

Method Validation. The optimized SPE-UHPLC-MS/MS method was validated according to the guidelines defined by the U.S. Food and Drug Administration (FDA) for bioanalytical method validation. ${ }^{21}$ Calibration curves were prepared in both solvent and blank urine at 12 concentration levels in the range $0.01-2000 \mu \mathrm{g} \mathrm{L}^{-1}$ by diluting individual stock solutions of standards $\left(1000 \mathrm{mg} \mathrm{L}^{-1}\right)$. Method specificity was evaluated by comparing the RT of authentic standards dissolved in solvent and blank urine. The limits of quantification (LOQs) were estimated in spiked urines as the lowest concentration that gives an average signal-to-noise $(\mathrm{S} / \mathrm{N})$ ratio above 10 , with accuracies varying from 80 to $120 \%$ of the theoretical value. LOQs were calculated by subtracting the analyte response observed in non-spiked blank urines. Intra- and interday precisions were evaluated by analyzing spiked urine samples at three concentration levels $(1,10$, and $\left.100 \mu \mathrm{g} \mathrm{L}^{-1}\right) 5$ times within the same day as well as on 3 consecutive days, respectively. Recoveries were determined in urine samples spiked at three concentration levels $\left(1,10\right.$, and $\left.100 \mu \mathrm{g} \mathrm{L}^{-1}\right)$, which were analyzed in triplicate. Matrix effects (MEs) were measured by comparing the analyte response of standards dissolved in solvent and urine at the same concentration level $\left(100 \mu \mathrm{g} \mathrm{L}^{-1}\right)$. 


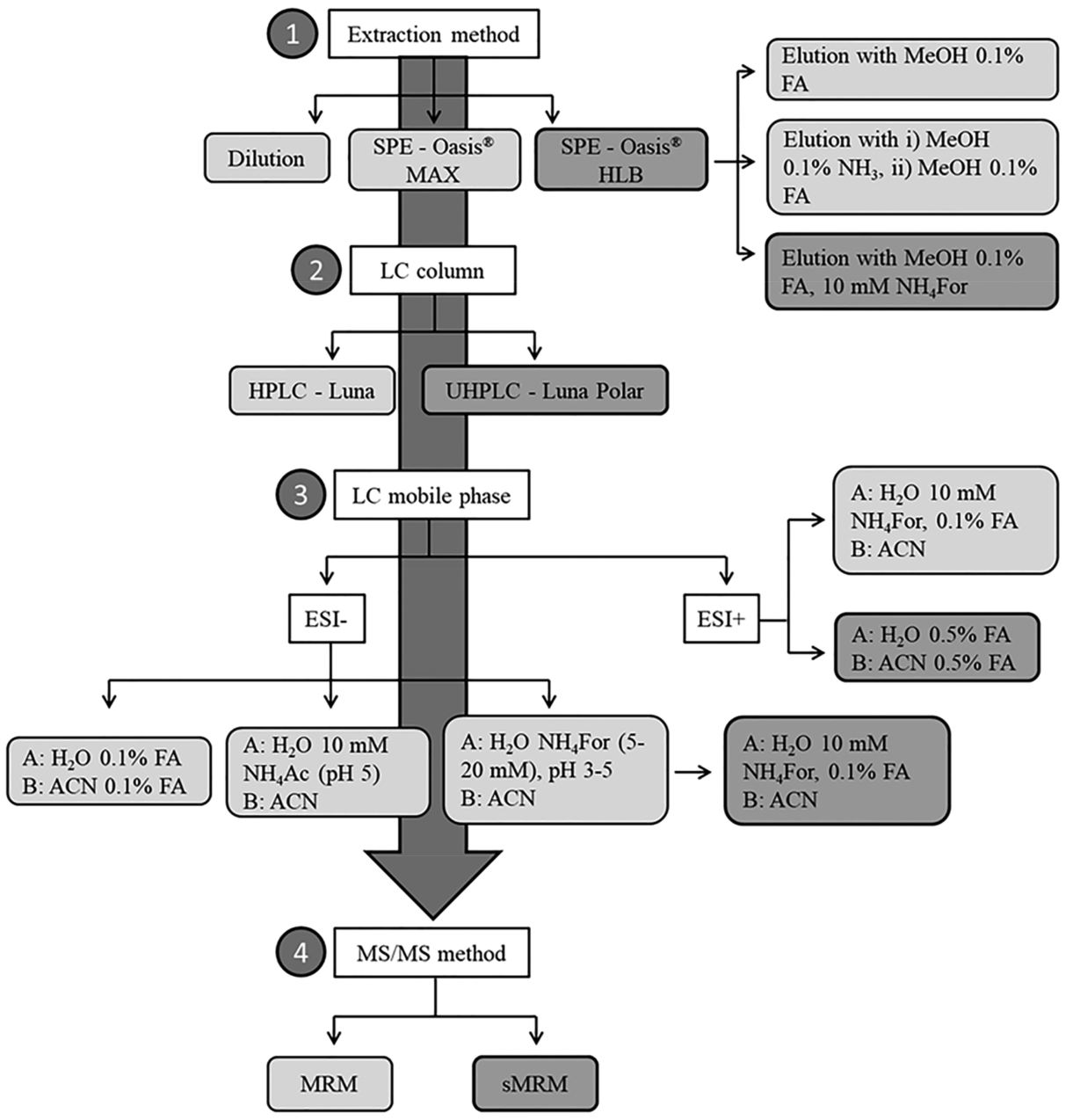

Figure 1. Flowchart showing the different steps considered for the optimization of the QDF approach.

\section{RESULTS AND DISCUSSION}

The QDF approach validated in this work is based on quantitative targeted nutrimetabolomic analysis of urine samples for comprehensive dietary assessment. To this end, we considered almost 350 dietary compounds and their host and microbial metabolites based on the knowhow of the research group and previous findings in metabolomic studies associated with the consumption of certain foods or dietary patterns (Table 1). ${ }^{22-48,49,50}$ These dietary compounds belong to different chemical classes, which were selected taking into consideration the potential biotransformations occurring after food intake and the availability of commercial standards, to obtain a representative overview of the food metabolome. Most of these monitored dietary markers ( 90\%) were (poly)phenolic compounds, including phenolic acids, hydroxybenzenes and hydroxybenzaldehydes, flavonoids, coumarins, stilbenoids, tyrosol derivatives, and enterolignans, as well as microbiota-derived urolithins and hydroxyphenylvalerolactones. In turn, phenolic acids can be further divided into several subclasses, such as hydroxybenzoic, hydroxyphenylacetic, hydroxycinnamic, and hydroxyphenylpropionic acids, while the flavonoid family comprises flavan-3-ols, flavanones, flavones, flavonols, isoflavones, dihydrochalcones, and anthocyanins (Table 1). Moreover, other non-polyphenolic analytes were also covered in this method, including glucosinolates, amino acid derivatives (e.g., peptides and $S$-allylcysteine), methylxanthines, alkaloids, and other miscellaneous com- pounds. To obtain accurate metabolic patterns associated with dietary habits, we carefully optimized and validated a metabolomic platform based on SPE-UHPLC-MS/MS operating in negative and positive ion modes, as schematized in Figure 1.

Optimization of the Urine Treatment Method. Dilution was first tested as the sample treatment method prior to targeted UHPLC-MS/MS profiling given the difficulty of optimizing an extraction protocol for the recovery of the entire set of dietary metabolites included in this study. The analysis of blank urine samples spiked with known concentrations of standards demonstrated that 10-fold dilution is the optimum dilution factor to minimize MEs provoked by the high salt content of this biofluid. However, this strategy yielded LOQs above $10 \mu \mathrm{g} \mathrm{L}^{-1}$ for almost all compounds monitored, which would significantly hinder the detection of these dietary markers in real urine samples. Accordingly, various SPE procedures were compared to the aim of increasing method sensitivity. First, preliminary experiments were conducted to select the most appropriate SPE sorbent. Reversed-phase resins are commonly employed for the cleanup of complex biological samples and the extraction of numerous dietary components, especially polyphenols. ${ }^{51}$ Nevertheless, the application of anion-exchange SPE has also been proposed to increase the recovery of acidic compounds, such as phase II sulfate-derived metabolites. In this work, we compared the performance of Oasis HLB and Oasis MAX plates for the 

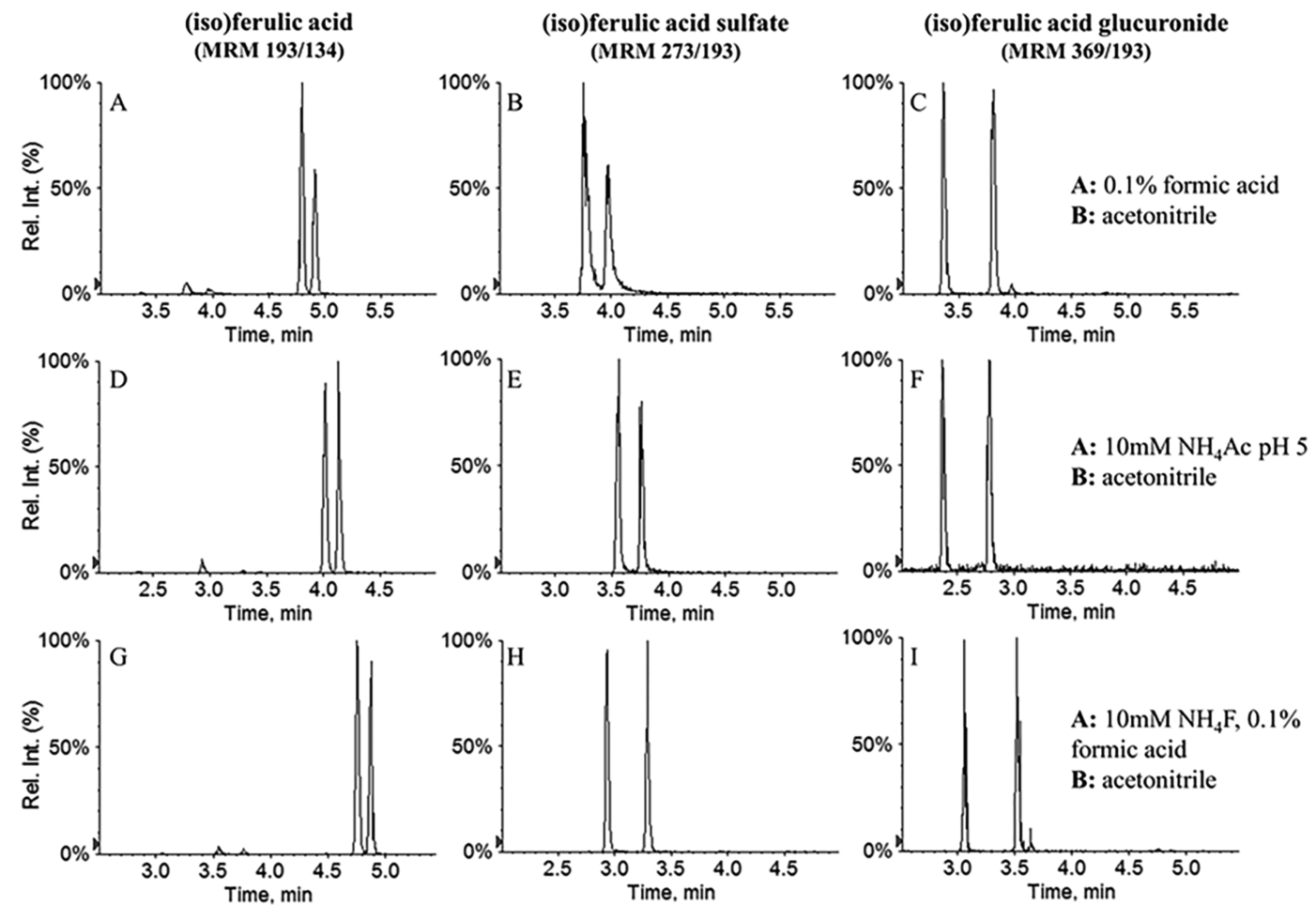

Figure 2. Representative chromatograms of (A, D, and G) ferulic/isoferulic acid, (B, E, and H) ferulic/isoferulic sulfate, and (C, F, and I) ferulic/ isoferulic glucuronide, using (A, B, and C) $0.1 \%$ formic acid, (D, E, and F) $10 \mathrm{mM}$ ammonium acetate at pH 5 , and $(\mathrm{G}, \mathrm{H}$, and I) $10 \mathrm{mM}$ ammonium formate and $0.1 \%$ formic acid as the aqueous mobile phase.

extraction of urinary dietary markers following previously optimized protocols. ${ }^{18,19}$ The use of MAX sorbents provided slightly higher recovery rates for a few flavan-3-ols (e.g., procyanidins B2, Dp3 and Dp4, and epigallocatechin) and some sulfate metabolites (e.g., curcumin $O$-sulfate and hesperetin 7-sulfate) (results not shown), but in general terms, reversed-phase SPE was best suited to the simultaneous extraction of different chemical classes of food compounds (extraction recovery ratios of HLB/MAX in the range of 0.06350; 15 times higher on average for HLB). Once the HLB plate had been selected, various solvents and SPE conditions were tested to maximize extraction recoveries, especially for sulfate metabolites poorly retained in this sorbent. For this purpose, we first tried the two-step method proposed by Khymenets et al. for sequential elution of glucuronide and sulfate species of epicatechin metabolites present in human breast milk using acidified and basic methanol, respectively. ${ }^{20}$ In the same vein, the utility of adding ammonium formate to SPE solvents to improve the extraction of polar compounds in reversed-phase resins was also checked. Similar results were obtained using these two SPE procedures (no significant differences tested by the $t$ test; $p>0.05$ ), with good recoveries for aglycone, glucuronide, and sulfate forms of food metabolites. Therefore, the SPE method based on the use of ammonium formate as a modifier was selected for further analyses as a result of its greater simplicity. Finally, we also evaluated the potential of introducing a pre-concentration step in this extraction protocol to increase method sensitivity. We first tried to reduce the total volume of the elution solvent below $1 \mathrm{~mL}$ (the volume of urine loaded into the SPE plate), but recovery rates were negatively affected. As experimentally checked, higher extraction efficiencies were achieved using 1.5 $\mathrm{mL}$ of methanol containing $0.1 \%$ formic acid and $10 \mathrm{mM}$ ammonium formate as the elution solvent. Alternatively, SPE extracts were taken to dryness using a vacuum concentrator and were then reconstituted in $100 \mu \mathrm{L}$ of mobile phase, thereby applying a 10 -fold pre-concentration factor. Interestingly, no substantial losses were observed during this evaporation step for any analyzed metabolite (no significant differences tested by the $t$ test; $p>0.05$ ), and furthermore, UHPLC peak shapes were significantly improved.

The extraction performance of this SPE method was excellent for most compounds detected by UHPLC-MS/MS in the negative acquisition mode as well as for anthocyanins, glucosinolates, methylxanthines, and some other metabolites determined under positive ionization (see the Analytical Validation of the QDF Method section). However, extraction efficiency was very low for some flavan-3-ols, such as procyanidins B2, Dp3 and Dp4, epigallocatechin, epicatechin gallate, and epigallocatechin gallate, probably because of the occurrence of strong interactions between these molecules and the SPE sorbent, as previously described. ${ }^{52}$ It is also worth noting that benzoic acid was not quantifiable using this extraction procedure as a result of the presence of some interfering compounds (checked in blank extracts). Consequently, all of these metabolites were discarded for the subsequent optimization of the UHPLC-MS/MS method. On the other hand, a few highly polar metabolites $(\mathrm{RT}<1 \mathrm{~min})$ showed low recovery rates as a result of their poor retention on reversed-phase resins. As an alternative, urine samples were complementarily analyzed after simple 10-fold dilution to expand the coverage of our targeted metabolomic approach toward the hydrophilic food metabolome, which comprises conventional markers of the intake of meat and fish [1methylhistidine, 3-methylhistidine, carnosine, anserine, Lcarnitine, creatinine, and trimethylamine $\mathrm{N}$-oxide (TMAO)], 
coffee (trigonelline and $N$-methylpyridinium), citrus fruits (proline betaine and 4-hydroxyproline betaine), alcoholic (ethyl glucuronide and ethyl sulfate) and sweetened (acesulfame $\mathrm{K}$ and cyclamate) beverages, or tobacco (nicotine and cotinine), among others.

Optimization of the Chromatographic Method. Separation conditions were optimized to achieve good resolution of metabolites listed in Table S2 of the Supporting Information. To this end, we employed a reversed-phase UHPLC column (Luna Omega Polar), which shows improved retention of polar metabolites. Given the different physicochemical properties of metabolites monitored in this study, two complementary UHPLC methods were developed for data acquisition in negative and positive ionization modes.

As a preliminary experiment, we employed similar mobile phases to those reported in previously published targeted approaches for urinary polyphenol profiling based on HPLCMS operating in the negative ion mode, consisting of water and acetonitrile both containing $0.1 \%$ formic acid. ${ }^{19}$ It is worth noting that the use of UHPLC considerably increased sensitivity and chromatographic separation compared to those obtained in HPLC columns, but poor resolution was observed for sulfate derivatives (Figure 2). To enhance the peak shape of these phase II metabolites, we tested the mobile phase proposed by Rotches-Ribalta et al. for the analysis of urinary resveratrol metabolites based on the addition of ammonium acetate to the aqueous phase to reduce the peak tailing of sulfate conjugates. ${ }^{15}$ Thus, we observed a better peak resolution using $10 \mathrm{mM}$ ammonium acetate $(\mathrm{pH} 5)$ and acetonitrile as aqueous and organic mobile phases, but in contrast, signal intensities were considerably reduced for most aglycone and glucuronide species (Figure 1). Because this loss of sensitivity was probably a consequence of ion suppression induced by this additive, we substituted ammonium acetate with ammonium formate, which is more volatile. As a result, even higher sensitivity to that observed without the addition of any modifier was obtained and a good peak resolution was maintained for sulfate metabolites (Figure 2). Then, different ammonium formate concentrations $(5-20 \mathrm{mM})$ and acidities ( $\mathrm{pH} 3-5,0.1 \%$ formic acid) were tested, and the best results were achieved using $10 \mathrm{mM}$ ammonium formate and $0.1 \%$ formic acid ( $\mathrm{pH}$ 3.3). To conclude, we also evaluated the separation performance of methanol as an organic mobile phase, but a considerable worsening of peak shapes was observed.

To optimize chromatographic conditions to be used in the positive MS ion mode, we first tried the mobile phases described above but broad chromatographic peaks were observed for anthocyanins. Then, different concentrations of formic acid, in the range of $0.1-1 \%$, were tested to increase the acidity of mobile phases, which is known to provide a better resolution for anthocyanins. ${ }^{53}$ Accordingly, the addition of $0.5 \%$ formic acid to both aqueous and organic mobile phases was proven to be the most suitable strategy for simultaneously analyzing anthocyanins, glucosinolates, methylxanthines, and other metabolites under positive electrospray ionization, as listed in Table S2 of the Supporting Information.

After the selection of the mobile phase composition, different chromatographic conditions were tested to achieve the maximum separation of urinary metabolites, especially for isomeric compounds. Various flow rates $\left(0.4-0.8 \mathrm{~mL} \mathrm{~min}^{-1}\right)$, column temperatures $\left(30-40{ }^{\circ} \mathrm{C}\right)$, and injection volumes $(2-$ $5 \mu \mathrm{L}$ ) were studied, and optimum results were obtained by delivering mobile phases at $0.5 \mathrm{~mL} \mathrm{~min}^{-1}$ and using $40{ }^{\circ} \mathrm{C}$ and $2 \mu \mathrm{L}$ as the column temperature and injection volume, respectively. In the negative ion mode, the optimized elution program begins with a slow gradient within $8 \mathrm{~min}$, ranging from 5 to $20 \%$ of the organic mobile phase, with the aim of obtaining a good separation of multiple phenolic acid isomers, such as hydroxybenzoic acids, dihydroxybenzoic acids, hydroxyphenylacetic acids, coumaric acids, and many others. Then, the proportion of organic solvent rapidly rises to $100 \%$ to elute more retained flavonoid metabolites. Under these chromatographic conditions, all metabolites were resolved within $10 \mathrm{~min}$, with a $14 \mathrm{~min}$ total run time, including washing and conditioning steps. On the other hand, dietary compounds analyzed in positive polarity were separated using a singlegradient program $(5-50 \% \mathrm{~B})$.

Optimization of the MS/MS Method. To maximize ionization efficiency and overall sensitivity, general MS parameters, such as source temperature, gas flow rates, and ionization voltage, were first optimized by evaluating peak areas and $\mathrm{S} / \mathrm{N}$ ratios in extracted ion chromatograms. Then, optimum MRM transitions and fragmentation conditions (i.e., DP, CE, and CXP) were selected by infusing standard solutions into the mass spectrometer using a syringe pump (Table S2 of the Supporting Information). For this, $500 \mu \mathrm{g} \mathrm{L}^{-1}$ individual solutions were prepared in a $1: 1(\mathrm{v} / \mathrm{v})$ mixture of acetonitrile and water containing $10 \mathrm{mM}$ ammonium formate and $0.1 \%$ formic acid for metabolites analyzed in the negative ion mode or in a 1:1 (v/v) acetonitrile/water mixture containing $0.5 \%$ formic acid for those ionized under positive polarity. Two MRM transitions were optimized for each analyte, with the most sensitive one for quantitation purposes and the other as a qualifier transition. However, as a result of the very large number of metabolites included in this study, two separate acquisition methods were created (i.e., quantitative and qualitative methods) to maximize the number of points across chromatographic peaks to obtain better peak detection and improve reproducibility. For the same reason, isomeric metabolites with common fragmentation patterns were grouped within a single transition. Additionally, the sMRM mode was employed instead of conventional MRM, which allows for the simultaneous monitoring of multiple transitions using RT windows. To establish these windows, individual standard solutions were injected into the UHPLCMS system to find their RTs, and RT windows were then estimated on the basis of peak widths and retention reproducibility (Table S2 of the Supporting Information).

Analytical Validation of the QDF Method. The QDF approach previously optimized on the basis of targeted metabolomic analysis of urine samples by SPE-UHPLCMS/MS was validated according to the FDA guidelines in terms of specificity, linearity, sensitivity, intra- and interday precision, recovery rate, and MEs (Table S3 of the Supporting Information). To evaluate method specificity, we compared the retention time of standard compounds in spiked urine samples to that observed when dissolved in the mobile phase. $\mathrm{RT}$ deviations $\left(\Delta \mathrm{RT}=\mathrm{RT}_{\text {sample }}-\mathrm{RT}_{\text {std }}\right)$ were below $0.04 \mathrm{~min}$ for all metabolites, thereby demonstrating the great stability of the UHPLC system. For most monitored compounds, calibration curves showed linear responses over 4-5 orders of magnitude in the studied concentration range $(0.01-1000$ $\left.\mu \mathrm{g} \mathrm{L}^{-1}\right)$, with correlation coefficients above 0.999 . However, these linear ranges were slightly reduced for some phenolic acids as a result of their higher LOQs as well as for some very 


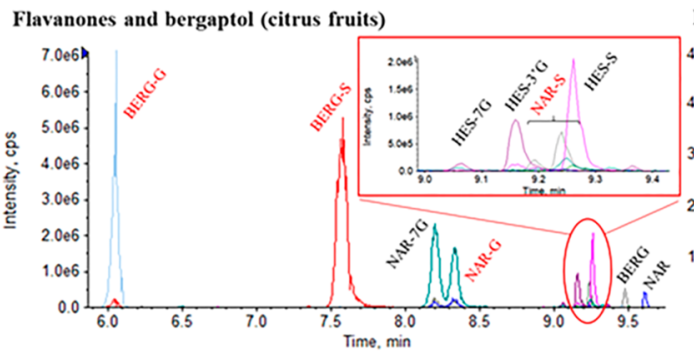

Isoflavones (soy and legumes)
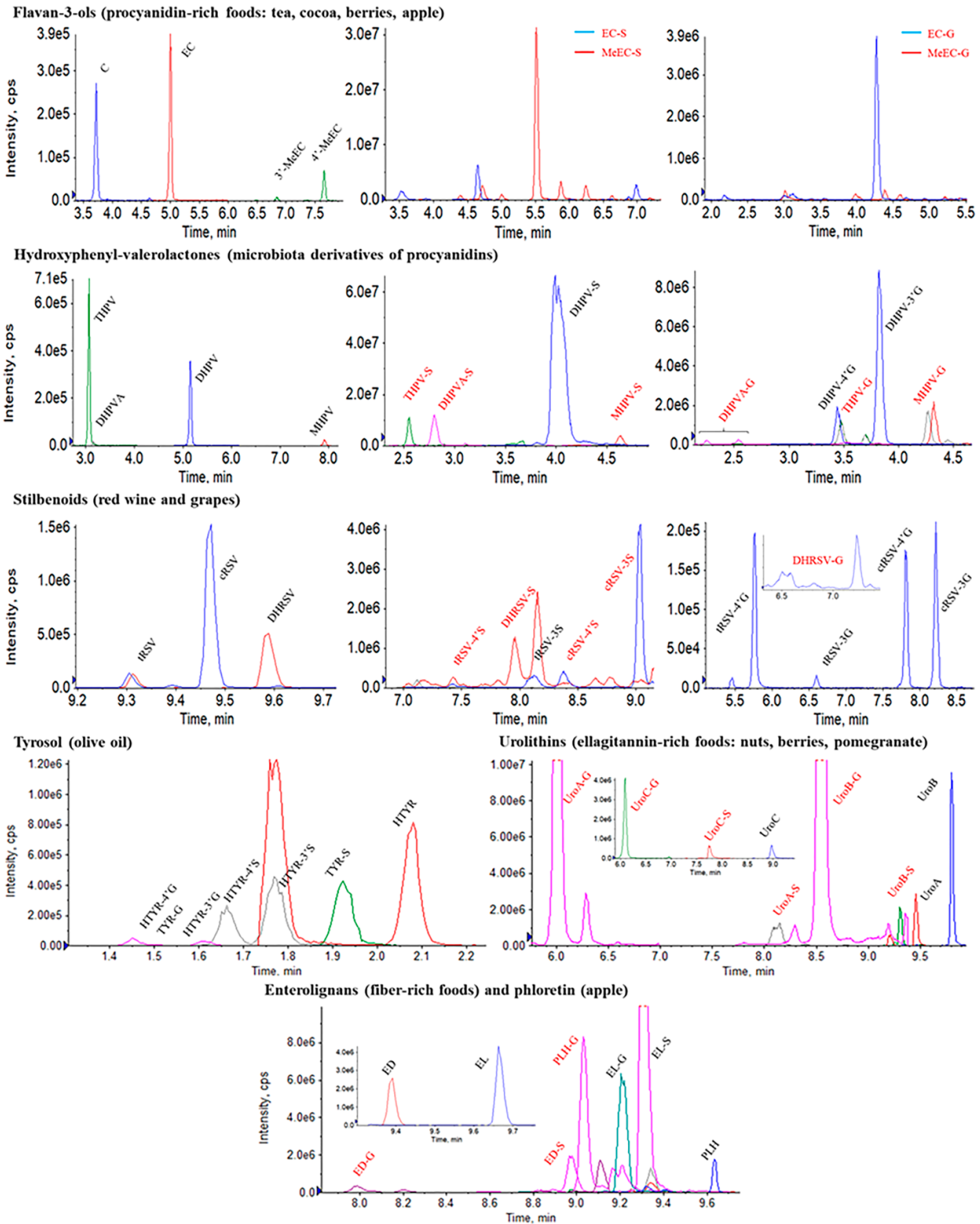

Figure 3. Extracted ion chromatograms acquired under negative ionization of representative metabolites present in urine samples. Metabolites labeled in red were identified in urine samples collected after acute dietary interventions by $\mathrm{MS}^{3}$.

polar metabolites detected in the positive ion mode $(\mathrm{RT}<1$ min) probably as a result of ion suppression effects (see Table S3 of the Supporting Information). The sensitivity of the method was estimated by computing the corresponding LOQs in spiked urine samples, which were calculated as the concentration needed to produce a chromatographic signal
10 times higher than the baseline noise. From the entire set of metabolites included in the method, $83 \%$ could be quantified at sub-parts per billion (ppb) levels, while 28 of the remaining metabolites displayed LOQs in the range $1-10 \mu \mathrm{g} \mathrm{L}^{-1}$. Only nine phenolic acids showed quantification limits above $10 \mu \mathrm{g}$ $\mathrm{L}^{-1}\left(11.5-40.0 \mu \mathrm{g} \mathrm{L}^{-1}\right)$. The results obtained were satisfactory 
for quantitating target compounds at concentrations usually found in urine samples. Instrumental precision was evaluated by analyzing blank urine samples spiked at three concentration levels, 5 times within the same day (intraday precision) as well as on 3 consecutive days (interday precision). Relative standard deviations (RSDs) varied in the ranges of 0.7-12.8 and $1.7-17.1 \%$ for the intra- and interday precision, respectively, suggesting that instrumental response is reproducible over a minimum period of 3 days. Recovery rates also met the FDA acceptance criteria, ranging from 80 to $120 \%$ for about $94 \%$ of the metabolites analyzed. Only a few flavonoid aglycones showed slightly lower extraction efficiencies (70.2$78.9 \%)$, while the SPE recovery of quercetin $3^{\prime}$-sulfate was unsatisfactory (10\%). For isotopically labeled ISs (i.e., ferulic acid- $1,2,3-{ }^{13} \mathrm{C}_{3}$, $p$-coumaric acid- $1,2,3-{ }^{13} \mathrm{C}_{3}$, catechin- $2,3,4-{ }^{13} \mathrm{C}_{3}$, and L-phenylalanine- ${ }^{15} \mathrm{~N}$ ), recoveries were in the range of $87-$ $104 \%$, thereby demonstrating the utility of this primary IS set for monitoring the efficiency of the extraction process. Finally, the ME was estimated to evaluate the performance of the sample treatment method and the UHPLC separation program for minimizing the impact of the urine matrix in the detection of dietary compounds. For this purpose, MS responses were compared for each standard dissolved in solvent and urine at the same concentration level. Overall, MEs were negligible for almost all compounds monitored (ME of 82.2-126\%), except for a few metabolites slightly affected by ion suppression (ME of $61.1-79.5 \%$ ), especially those analyzed in positive ion mode after simple dilution (e.g., carnosine and 3-methylhistidine). Therefore, given the good recovery rates and low MEs obtained, the method could be applied to real urine samples using calibration curves directly prepared in solvent, thereby simplifying the analytical workflow.

In comparison to previous validated methods based on targeted metabolomics, the QDF approach provides comparable linearity and intra- and interday precision. ${ }^{14-16}$ However, sensitivity and ME were significantly improved thanks to the application of a reliable SPE protocol and further pre-concentration step to simultaneously extract the urinary food metabolome. This is especially remarkable when comparing to high-throughput methods based on direct analysis of diluted urine samples.

Application to Real Urine Samples: Identification of Novel Conjugate Metabolites. After the optimization of the SPE method, chromatographic conditions, and MS detection parameters, the developed QDF metabolomic platform was applied to urine samples collected after acute dietary interventions with various foods with the aim of increasing the number of biologically interesting phase II metabolites for which commercial standards are not available to enlarge the coverage of our dietary fingerprinting method. For this purpose, product ion scan experiments $\left(\mathrm{MS}^{2}\right)$ were conducted using predicted nominal masses of these expected metabolites and those peaks showing neutral losses of $176 \mathrm{Da}$ (i.e., glucuronide conjugates) or $80 \mathrm{Da}$ (i.e., sulfate conjugates) were subjected to $\mathrm{MS}^{3}$ fragmentation of the corresponding aglycone. Using this method, we successfully identified 127 urinary compounds associated with the consumption of orange, apple, grapefruit, red wine, green tea, coffee, soy, walnuts, and whole grain bread (Table S4 of the Supporting Information). To include these potential dietary markers in our targeted metabolomic approach, MRM transitions and fragmentation parameters were estimated on the basis of results obtained with available standards of other metabolites.
As a rule, we observed that DPs and CXPs optimized for phase II conjugates with available authentic standards were very similar to those employed for their aglycone counterparts. In contrast, glucuronide and sulfate metabolites needed slightly higher CEs to yield significant fragmentation than the corresponding aglycone forms. Thus, MRM detection of conjugates identified in urine samples was accomplished by applying the following parameters: (i) quantifier MRM transition, $\mathrm{DP}_{\text {conjugate }}=\mathrm{DP}_{\text {aglycone, }} \mathrm{CXP}_{\text {conjugate }}=\mathrm{CXP}_{1 \text {-aglycone, }}$ and $\mathrm{CE}_{\text {conjugate }}=\mathrm{CE}_{1 \text {-aglycone }}+5 \mathrm{~V}$; (ii) qualifier MRM transition, $\mathrm{DP}_{\text {conjugate }}=\mathrm{DP}_{\text {aglycone }}, \mathrm{CXP}_{\text {conjugate }}=\mathrm{CXP}_{1 \text {-aglycone, }}$ and $\mathrm{CE}_{\text {conjugate }}=\mathrm{CE}_{1 \text {-aglycone }}+15 \mathrm{~V}$ (Table $\mathrm{S} 2$ of the Supporting Information). Thus, this optimized analytical strategy enables the detection of these novel phase II metabolites in a "semi-quantitative" manner, by either quantifying them using calibration curves built with standards of structurally similar compounds (i.e., the corresponding aglycones or isomeric phase II metabolites, if available) or measuring their peak area (relative to the IS).

Using this QDF methodology, about 350 compounds representative of specific food class consumption could be successfully measured in urine samples, thereby enabling a comprehensive assessment of dietary habits. The analysis of urine samples collected after dietary interventions with various plant-derived foods showed a significant increase in the urinary levels of phenolic acids. The most abundant free phenolic compounds were 4-hydroxybenzoic acid, hippuric acids, hydroxyphenylacetic acids, and homovanillic acid, while among phase II metabolites, sulfate conjugates were very concentrated in urine, largely surpassing the concentration levels of the corresponding aglycone forms. Furthermore, the consumption of specific foods was reflected in the excretion of the following polyphenol metabolites: flavanone metabolites (i.e., naringenin and hesperetin) were associated with orange intake; isoflavones (i.e., daidzein and genistein) were associated with soy; dihydrochalcones (i.e., phloretin) were associated with apple; furanocoumarins (i.e., bergaptol) were associated with grapefruit; prenylflavonoids (i.e., isoxanthohumol) were associated with beer; flavan-3-ols were associated with epicatechin-rich foods (e.g., apple and tea); flavones (i.e., apigenin and luteolin) and flavonols (i.e., quercetin, kaempferol, and isorhamnetin) were associated with fruits and vegetables; enterolignans (i.e., enterolactone and enterodiol) were associated with fiber-rich foods; stilbenoids (i.e., resveratrol) were associated with red wine; and tyrosol metabolites were associated with olive oil, as summarized in Figures 3 and 4. These food compounds underwent extensive metabolization by phase II enzymes, thereby yielding a myriad of glucuronide, sulfate, and sulfoglucuronide conjugates in urine. However, the aglycone forms of some of these flavonoids could also be detected at quantifiable concentrations in the urine samples analyzed in this study, such as naringenin, bergaptol, phloretin, isoxanthohumol, daidzein, and genistein, among others. The optimized method also enabled the detection of various metabolites derived from microbiota biotransformation of some scarcely absorbed food components, including hydroxyphenylvalerolactones produced in the ring-fission metabolism of catechins (apple and tea), urolithins coming from ellagitannins (walnut), and aminophenol-derived metabolites [i.e., 2-aminophenol and $\mathrm{N}$-(2hydroxyphenyl)acetamide] generated by microbial degradation of bezoxazinoids (whole grain). These compounds were mainly detected in urine samples as phase II metabolites 


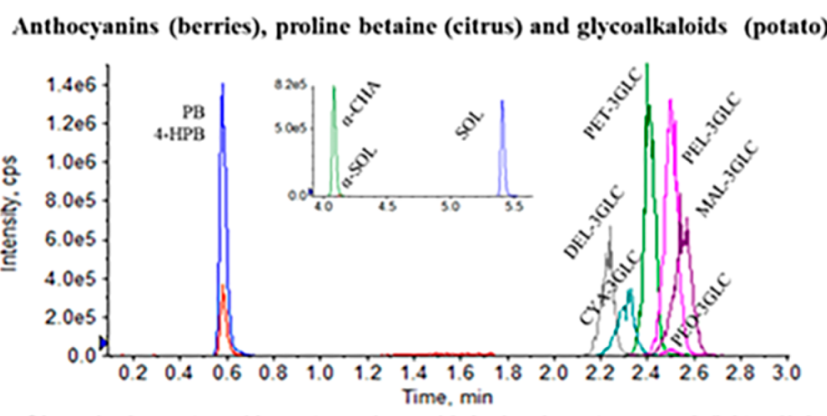

Glucosinolates (cruciferous), amino acid derivatives (meat and fish), allyl-

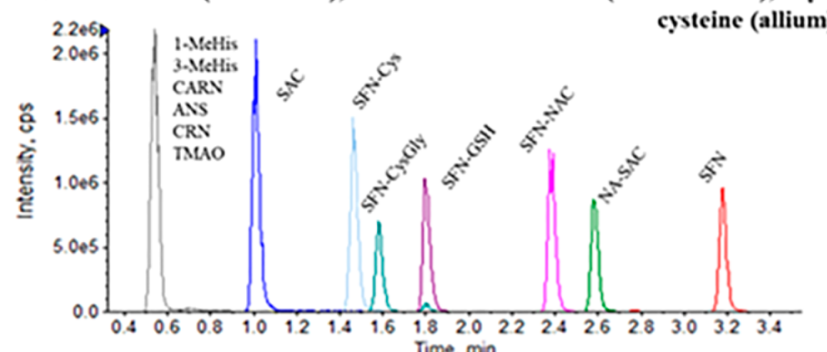

Methylxanthines, diketopyperazines and pyridinium derivatives (coffec,

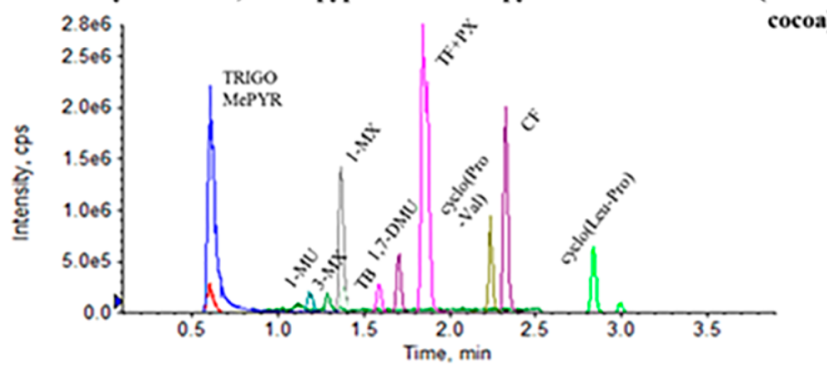

Figure 4. Extracted ion chromatograms acquired under positive ionization of representative metabolites present in urine samples.

(sulfated conjugates for valerolactones and benzoxazinoids and glucuronidated conjugates for urolithins), but the corresponding aglycones were also found. Additionally, the complementary analysis of urines in the positive ion mode expanded the method coverage toward the determination of anthocyanins, methylxanthines, glucosinolates, glycoalkaloids, and S-allylcysteine derivates, suitable dietary markers of the consumption of berries; coffee, cocoa, and tea; cruciferous vegetables (e.g., cabbage and broccoli); potato; and vegetables from the Allium genus (e.g., onion and garlic), respectively.

In conclusion, it should be noted that the QDF methodology optimized here shows a great potential for comprehensive assessment of dietary patterns and lifestyles, by simultaneously analyzing about 350 urinary metabolic biomarkers associated with the consumption of plant-derived foods, animal foods, and alcoholic and non-alcoholic beverages, as well as smoking status. This is a first step toward the development of precision nutrition and medicine, because it allows for quantification of the human diet in a robust and objective way, considering its metabolic complexity and interindividual variability.

\section{ASSOCIATED CONTENT}

\section{S Supporting Information}

The Supporting Information is available free of charge on the ACS Publications website at DOI: 10.1021/acs.jafc.8b07023.

Commercial source of chemicals and standards employed in the study, detailed description of foods employed in dietary interventions (Table S1), optimized MRM transitions and fragmentation conditions (Table S2), summary of validation parameters for 220 dietary metabolites with authentic standards (Table S3), and UHPLC-MS ${ }^{2} / \mathrm{MS}^{3}$-based identification of 127 phase II metabolites in urine samples collected after acute dietary interventions (Table S4) (PDF)

\section{AUTHOR INFORMATION}

\section{Corresponding Author}

*Telephone: +34-934034840. Fax: +34-934035931. E-mail: candres@ub.edu.

\section{ORCID}

Raúl González-Domínguez: 0000-0002-7640-8833

Paul A. Kroon: 0000-0002-9805-6947

Cristina Andrés-Lacueva: 0000-0002-8494-4978

\section{Author Contributions}

Raúl González-Domínguez, Mireia Urpi-Sarda, and Cristina Andrés-Lacueva designed the research. Raúl GonzálezDominguez and Olga Jauregui conducted the experimental analyses. Raúl González-Dominguez analyzed the data and wrote the first draft of the manuscript. Paul W. Needs and Paul A. Kroon provided in-house synthesized standards for 35 metabolites (as detailed in the Supporting Information). All authors read and approved the final manuscript. Cristina Andrés-Lacueva has primary responsibility for final content.

\section{Funding}

This work was supported by the Spanish Ministry of Economy and Competitiveness (MINECO) together with the Joint Programming Initiative "A Healthy Diet for a Healthy Life" (PCIN-2015-238), the CIBERfes (co-funded by the FEDER Program from the European Union), the Generalitat de Catalunya's Agency AGAUR (2017 SGR 1546), and the EIT Health Project COOK2HEALTH. Raúl González-Domínguez thanks the "Juan de la Cierva" program from MINECO (FJCI2015-26590). Paul W. Needs and Paul A. Kroon gratefully acknowledge the support of the Biotechnology and Biological Sciences Research Council (BBSRC) through an Institute Strategic Programme (Food Innovation and Health, BB/ $\mathrm{R} 012512 / 1)$ and its constituent project(s) BBS/E/F/ 000PR10343 and BBS/E/F/000PR10346.

\section{Notes}

The authors declare no competing financial interest.

\section{REFERENCES}

(1) Poslusna, K.; Ruprich, J.; de Vries, J. H.; Jakubikova, M.; van't Veer, P. Misreporting of energy and micronutrient intake estimated by food records and $24 \mathrm{~h}$ recalls, control and adjustment methods in practice. Br. J. Nutr. 2009, 101, S73-85.

(2) Ulaszewska, M. M.; Weinert, C. H.; Trimigno, A.; Portmann, R.; Andres-Lacueva, C.; Badertscher, R.; Brennan, L.; Brunius, C.; Bub, A.; Capozzi, F.; Cialiè Rosso, M.; Cordero, C. E.; Daniel, H.; Durand, S.; Egert, B.; Ferrario, P. G.; Feskens, E. J. M.; Franceschi, P.; GarciaAloy, M.; Giacomoni, F.; Giesbertz, P.; González-Domínguez, R.; Hanhineva, K.; Hemeryck, L. Y.; Kopka, J.; Kulling, S. E.; Llorach, R.; Manach, C.; Mattivi, F.; Migné, C.; Münger, L. H.; Ott, B.; Picone, G.; Pimentel, G.; Pujos-Guillot, E.; Riccadonna, S.; Rist, M. J.; Rombouts, C.; Rubert, J.; Skurk, T.; Sri Harsha, P. S. C.; Van Meulebroek, L.; Vanhaecke, L.; Vázquez-Fresno, R.; Wishart, D.; Vergères, G. Nutrimetabolomics: An integrative action for metabolomic analyses in human nutritional studies. Mol. Nutr. Food Res. 2019, 63, No. 1800384. 
(3) Scalbert, A.; Brennan, L.; Manach, C.; Andres-Lacueva, C.; Dragsted, L. O.; Draper, J.; Rappaport, S. M.; van der Hooft, J. J.; Wishart, D. S. The food metabolome: A window over dietary exposure. Am. J. Clin. Nutr. 2014, 99, 1286-1308.

(4) Medina-Remón, A.; Tresserra-Rimbau, A.; Arranz, S.; Estruch, R.; Lamuela-Raventos, R. M. Polyphenols excreted in urine as biomarkers of total polyphenol intake. Bioanalysis 2012, 4, 27052713.

(5) Manach, C.; Donovan, J. L. Pharmacokinetics and metabolism of dietary flavonoids in humans. Free Radical Res. 2004, 38, 771-785.

(6) Selma, M. V.; Espin, J. C.; Tomas-Barberan, F. A. Interaction between phenolics and gut microbiota: Role in human health. J. Agric. Food Chem. 2009, 57, 6485-6501.

(7) Brouwer-Brolsma, E. M.; Brennan, L.; Drevon, C. A.; van Kranen, H.; Manach, C.; Dragsted, L. O.; Roche, H. M.; AndresLacueva, C.; Bakker, S. J. L.; Bouwman, J.; Capozzi, F.; De Saeger, S.; Gundersen, T. E.; Kolehmainen, M.; Kulling, S. E.; Landberg, R.; Linseisen, J.; Mattivi, F.; Mensink, R. P.; Scaccini, C.; Skurk, T.; Tetens, I.; Vergeres, G.; Wishart, D. S.; Scalbert, A.; Feskens, E. J. M. Combining traditional dietary assessment methods with novel metabolomics techniques: Present efforts by the Food Biomarker Alliance. Proc. Nutr. Soc. 2017, 76, 619-627.

(8) Spencer, J. P. E.; Abd El Mohsen, M. M.; Minihane, A.-M.; Mathers, J. C. Biomarkers of the intake of dietary polyphenols: Strengths, limitations and application in nutrition research. Br. J. Nutr. 2008, 99, 12-22.

(9) Saha, S.; Hollands, W.; Needs, P. W.; Ostertag, L. M.; de Roos, B.; Duthie, G. G.; Kroon, P. A. Human O-sulfated metabolites of $(-)$-epicatechin and methyl-(-)-epicatechin are poor substrates for commercial aryl-sulfatases: Implications for studies concerned with quantifying epicatechin bioavailability. Pharmacol. Res. 2012, 65, 592602.

(10) Gu, L.; Laly, M.; Chang, H. C.; Prior, R. L.; Fang, N.; Ronis, M. J.; Badger, T. M. Isoflavone conjugates are underestimated in tissues using enzymatic hydrolysis. J. Agric. Food Chem. 2005, 53, 68586863.

(11) Quifer-Rada, P.; Martínez-Huélamo, M.; Lamuela-Raventos, R. $M$. Is enzymatic hydrolysis a reliable analytical strategy to quantify glucuronidated and sulfated polyphenol metabolites in human fluids? Food Funct. 2017, 8, 2419-2424.

(12) Redeuil, K.; Smarrito-Menozzi, C.; Guy, P.; Rezzi, S.; Dionisi, F.; Williamson, G.; Nagy, K.; Renouf, M. Identification of novel circulating coffee metabolites in human plasma by liquid chromatography-mass spectrometry. J. Chromatogr. A 2011, 1218, 4678-4688.

(13) Feliciano, R. P.; Boeres, A.; Massacessi, L.; Istas, G.; Ventura, M. R.; Nunes Dos Santos, C.; Heiss, C.; Rodriguez-Mateos, A. Identification and quantification of novel cranberry-derived plasma and urinary (poly)phenols. Arch. Biochem. Biophys. 2016, 599, 31-41.

(14) Feliciano, R. P.; Mecha, E.; Bronze, M. R.; Rodriguez-Mateos, A. Development and validation of a high-throughput micro solidphase extraction method coupled with ultra-high-performance liquid chromatography-quadrupole time-of-flight mass spectrometry for rapid identification and quantification of phenolic metabolites in human plasma and urine. J. Chromatogr. A 2016, 1464, 21-31.

(15) Rotches-Ribalta, M.; Urpi-Sarda, M.; Llorach, R.; BotoOrdoñez, M.; Jauregui, O.; Chiva-Blanch, G.; Perez-Garcia, L.; Jaeger, W.; Guillen, M.; Corella, D.; Tinahones, J. J.; Estruch, R.; Andres-Lacueva, C. Gut and microbial resveratrol metabolite profiling after moderate long-term consumption of red wine versus dealcoholized red wine in humans by an optimized ultra-high-pressure liquid chromatography tandem mass spectrometry method. J. Chromatogr. A 2012, 1265, 105-113.

(16) Garcia-Villalba, R.; Espin, J. C.; Tomas-Barberan, F. A. Chromatographic and spectroscopic characterization of urolithins for their determination in biological samples after the intake of foods containing ellagitannins and ellagic acid. J. Chromatogr. A 2016, 1428, $162-175$.

(17) Khymenets, O.; Crespo, M. C.; Dangles, O.; Rakotomanomana, N.; Andres-Lacueva, C.; Visioli, F. J. Human hydroxytyrosol's absorption and excretion from a nutraceutical. J. Funct. Foods 2016, 23, 278-282.

(18) Zamora-Ros, R.; Rabassa, M.; Cherubini, A.; Urpi-Sarda, M.; Llorach, R.; Bandinelli, S.; Ferrucci, L.; Andres-Lacueva, C. Comparison of $24-\mathrm{h}$ volume and creatinine-corrected total urinary polyphenol as a biomarker of total dietary polyphenols in the Invecchiare InCHIANTI study. Anal. Chim. Acta 2011, 704, 110115.

(19) Urpi-Sarda, M.; Monagas, M.; Khan, N.; Llorach, R.; LamuelaRaventós, R. M.; Jáuregui, O.; Estruch, R.; Izquierdo-Pulido, M.; Andrés-Lacueva, C. Targeted metabolic profiling of phenolics in urine and plasma after regular consumption of cocoa by liquid chromatography-tandem mass spectrometry. J. Chromatogr. A 2009, 1216, 7258-7267.

(20) Khymenets, O.; Rabassa, M.; Rodríguez-Palmero, M.; RiveroUrgell, M.; Urpi-Sarda, M.; Tulipani, S.; Brandi, P.; Campoy, C.; Santos-Buelga, C.; Andres-Lacueva, C. Dietary epicatechin is available to breastfed infants through human breast milk in the form of host and microbial metabolites. J. Agric. Food Chem. 2016, 64, 5354-5360.

(21) U.S. Department of Health and Human Services, Food and Drug Administration (FDA). Bioanalytical Method Validation. Guidance for Industry; FDA: Silver Spring, MD, 2001.

(22) Crozier, A.; Del Rio, D.; Clifford, M. N. Bioavailability of dietary flavonoids and phenolic compounds. Mol. Aspects Med. 2010, 31, 446-467.

(23) Rothwell, J. A.; Madrid-Gambin, F.; Garcia-Aloy, M.; AndresLacueva, C.; Logue, C.; Gallagher, A. M.; Mack, C.; Kulling, S. E.; Gao, Q.; Praticò, G.; Dragsted, L. O.; Scalbert, A. Biomarkers of intake for coffee, tea, and sweetened beverages. Genes Nutr. 2018, 13, 15 .

(24) Michielsen, C. C. J. R.; Almanza-Aguilera, E.; Brouwer-Brolsma, E. M.; Urpi-Sarda, M.; Afman, L. A. Biomarkers of food intake for cocoa and liquorice (products): A systematic review. Genes Nutr. 2018, 13, 22.

(25) Angelino, D.; Cossu, M.; Marti, A.; Zanoletti, M.; Chiavaroli, L.; Brighenti, F.; Del Rio, D.; Martini, D. Bioaccessibility and bioavailability of phenolic compounds in bread: A review. Food Funct. 2017, 8, 2368-2393.

(26) Bolling, B. W.; Chen, C. Y.; McKay, D. L.; Blumberg, J. B. Tree nut phytochemicals: Composition, antioxidant capacity, bioactivity, impact factors. A systematic review of almonds, Brazils, cashews, hazelnuts, macadamias, pecans, pine nuts, pistachios and walnuts. Nutr. Res. Rev. 2011, 24, 244-275.

(27) Singh, B.; Singh, J. P.; Shevkani, K.; Singh, N.; Kaur, A. Bioactive constituents in pulses and their health benefits. J. Food Sci. Technol. 2017, 54, 858-870.

(28) Boskou, D.; Blekas, G.; Tsimidou, M. Phenolic compounds in olive oil and olives. Curr. Top. Nutraceutical Res. 2005, 3, 125-136.

(29) Del Rio, D.; Borges, G.; Crozier, A. Berry flavonoids and phenolics: Bioavailability and evidence of protective effects. Br. J. Nutr. 2010, 104, S67-S90.

(30) Rana, S.; Bhushan, S. Apple phenolics as nutraceuticals: Assessment, analysis and application. J. Food Sci. Technol. 2016, 53, $1727-1738$

(31) Zamora-Ros, R.; Knaze, V.; Rothwell, J. A.; Hémon, B.; Moskal, A.; Overvad, K.; Tjønneland, A.; Kyrø, C.; Fagherazzi, G.; BoutronRuault, M. C.; Touillaud, M.; Katzke, V.; Kühn, T.; Boeing, H.; Förster, J.; Trichopoulou, A.; Valanou, E.; Peppa, E.; Palli, D.; Agnoli, C.; Ricceri, F.; Tumino, R.; de Magistris, M. S.; Peeters, P. H.; Buenode-Mesquita, H. B.; Engeset, D.; Skeie, G.; Hjartåker, A.; Menéndez, V.; Agudo, A.; Molina-Montes, E.; Huerta, J. M.; Barricarte, A.; Amiano, P.; Sonestedt, E.; Nilsson, L. M.; Landberg, R.; Key, T. J.; Khaw, K. T.; Wareham, N. J.; Lu, Y.; Slimani, N.; Romieu, I.; Riboli, E.; Scalbert, A. Dietary polyphenol intake in Europe: The European Prospective Investigation into Cancer and Nutrition (EPIC) study. Eur. J. Nutr. 2016, 55, 1359-1375.

(32) Landete, J. M. Plant and mammalian lignans: A review of source, intake, metabolism, intestinal bacteria and health. Food Res. Int. 2012, 46, 410-424. 
(33) Gürdeniz, G.; Jensen, M. G.; Meier, S.; Bech, L.; Lund, E.; Dragsted, L. O. Detecting beer intake by unique metabolite patterns. J. Proteome Res. 2016, 15, 4544-4556.

(34) Curini, M.; Cravotto, G.; Epifano, F.; Giannone, G. Chemistry and biological activity of natural and synthetic prenyloxycoumarins. Curr. Med. Chem. 2006, 13, 199-222.

(35) Pimpão, R. C.; Dew, T.; Figueira, M. E.; McDougall, G. J.; Stewart, D.; Ferreira, R. B.; Santos, C. N.; Williamson, G. Urinary metabolite profiling identifies novel colonic metabolites and conjugates of phenolics in healthy volunteers. Mol. Nutr. Food Res. 2014, 58, 1414-1425.

(36) de Ferrars, R. M.; Cassidy, A.; Curtis, P.; Kay, C. D. Phenolic metabolites of anthocyanins following a dietary intervention study in post-menopausal women. Mol. Nutr. Food Res. 2014, 58, 490-502.

(37) Beckmann, M.; Lloyd, A. J.; Haldar, S.; Seal, C.; Brandt, K.; Draper, J. Hydroxylated phenylacetamides derived from bioactive benzoxazinoids are bioavailable in humans after habitual consumption of whole grain sourdough rye bread. Mol. Nutr. Food Res. 2013, 57, 1859-1873.

(38) Mithen, R. F. Glucosinolates and their degradation products. Adv. Bot. Res. 2001, 35, 213-232.

(39) Lanzotti, V.; Scala, F.; Bonanomi, G. Compounds from Allium species with cytotoxic and antimicrobial activity. Phytochem. Rev. 2014, 13, 769-791.

(40) Sucha, L.; Tomsik, P. The steroidal glycoalkaloids from Solanaceae: Toxic effect, antitumour activity and mechanism of action. Planta Med. 2016, 82, 379-387.

(41) Ashihara, H.; Ludwig, I. A.; Katahira, R.; Yokota, T.; Fujimura, T.; Crozier, A. Trigonelline and related nicotinic acid metabolites: Occurrence, biosynthesis, taxonomic considerations, and their roles in planta and in human health. Phytochem. Rev. 2015, 14, 765-798.

(42) Dragsted, L. O. Biomarkers of meat intake and the application of nutrigenomics. Meat Sci. 2010, 84, 301-307.

(43) Guasch-Ferré, M.; Bhupathiraju, S. N.; Hu, F. B. Use of metabolomics in improving assessment of dietary intake. Clin. Chem. 2018, 64, 82-98.

(44) Seok, Y. J.; Her, J. Y.; Kim, Y. G.; Kim, M. Y.; Jeong, S. Y.; Kim, M. K.; Lee, J. Y.; Kim, C. I.; Yoon, H. J.; Lee, K. G. Furan in thermally processed foods-A review. Toxicol. Res. 2015, 31, 241-253.

(45) Joshi, A. D.; Kim, A.; Lewinger, J. P.; Ulrich, C. M.; Potter, J. D.; Cotterchio, M.; Le Marchand, L.; Stern, M. C. Meat intake, cooking methods, dietary carcinogens, and colorectal cancer risk: Findings from the Colorectal Cancer Family Registry. Cancer Med. 2015, 4, 936-952.

(46) Armer, J. M.; Gunawardana, L.; Allcock, R. L. The performance of alcohol markers including ethyl glucuronide and ethyl sulphate to detect alcohol use in clients in a community alcohol treatment programme. Alcohol Alcohol. 2017, 52, 29-34.

(47) Lang, R.; Lang, T.; Bader, M.; Beusch, A.; Schlagbauer, V.; Hofmann, T. High-throughput quantitation of proline betaine in foods and suitability as a valid biomarker for citrus consumption. J. Agric. Food Chem. 2017, 65, 1613-1619.

(48) Benowitz, N. L.; Hukkanen, J.; Jacob, P., III Nicotine chemistry, metabolism, kinetics and biomarkers. In Nicotine Psycopharmacology. Handbook of Experimental Pharmacology; Henningfield, J. E., London, E. D., Pogun, S., Eds.; Springer-Verlag: Berlin, Germany, 2009; Vol. 192, pp 29-60, DOI: 10.1007/978-3540-69248-5 2.

(49) Kalaras, M. D.; Richie, J. P.; Calcagnotto, A.; Beelman, R. B. Mushrooms: A rich source of the antioxidants ergothioneine and glutathione. Food Chem. 2017, 233, 429-433.

(50) Regueiro, J.; Vallverdú-Queralt, A.; Simal-Gándara, J.; Estruch, R.; Lamuela-Raventós, R. M. Urinary tartaric acid as a potential biomarker for the dietary assessment of moderate wine consumption: A randomised controlled trial. Br. J. Nutr. 2014, 111, 1680-1685.

(51) Ajila, C. M.; Brar, S. K.; Verma, M.; Tyagi, R. D.; Godbout, S.; Valéro, J. R. Extraction and analysis of polyphenols: Recent trends. Crit. Rev. Biotechnol. 2011, 31, 227-249.
(52) Muñoz-González, I.; Sánchez-Patán, F.; Jiménez-Girón, A.; Cueva, C.; Monagas, M.; Martín-Álvarez, P. J.; Moreno-Arribas, M. V.; Bartolomé, B. Evaluation of SPE as preparative technique for the analysis of phenolic metabolites in human feces. Food Anal. Methods 2014, 7, 844-853.

(53) Valls, J.; Millán, S.; Martí, M. P.; Borràs, E.; Arola, L. Advanced separation methods of food anthocyanins, isoflavones and flavanols. $J$. Chromatogr. A 2009, 1216, 7143-7172. 